\title{
ASSESSMENT OF THE BREAST MASSES WITH DIAGNOSTIC MAMMOGRAPHY AND FNAC CORRELATION
}

\author{
Varsha Rathi ${ }^{1}$, Kalyani Patankar ${ }^{2}$
}

${ }^{1}$ Associate Professor, Department of Radiodiagnosis, Indira Gandhi Government Medical College, Nagpur, Maharashtra. ${ }^{2}$ Senior Resident, Department of Radiodiagnosis, Indira Gandhi Government Medical College, Nagpur, Maharashtra.

\begin{tabular}{l} 
ABSTRACT \\
\hline OBJECTIVES \\
Diagnostic mammography is the basic imaging study employed to evaluate breast abnormalities. Our objective was to assess the \\
role of diagnostic mammography in characterizing the breast lumps in correlation with cytopathology.
\end{tabular}

\section{STUDY DESIGN}

This prospective study of 63 patients of breast lumps and related complaints in the age group of 18-65 years, was done over a period of two years. Two standard radiological views Craniocaudal (CC) and Mediolateral Oblique (MLO) were taken. Additional views and ultrasound was done as and when required. Reporting of mammograms was done using standard ACR BIRADS $4^{\text {th }}$ edition (2003) Lexicon followed by cytopathological correlation.

\section{RESULTS}

In 63 patients with 70 lesions, 44 were proved cytopathological benign and 19 were malignant; 3 male patients were also included. Lump was the commonest presenting complaint. Left breast and superolateral quadrant with the axillary tail region was more frequently affected. Infiltrating ductal $\mathrm{Ca}(17.14 \%)$ was commonest malignant lesion and fibroadenoma the commonest benign lesion (30\%); 21 patients were categorized in BIRADS 3, 19 in BIRADS 1, 6 in BIRADS 2, 12 in BIRADS 4 and 5 in BIRADS 5 categories. In BIRADS 1, 2, 3 which were benign category, 43 were true negative, but 3 patients were pathologically malignant and hence false negative. Similarly, out of 17 malignant cases in BIRADS 4 and 5, 1 was false positive and 16 proved to be true positive. The statistical analysis was done and parameters calculated.

\section{CONCLUSION}

Diagnostic mammography is highly sensitive and accurate in detection and characterization of breast lumps, especially the malignant and the ACR-BIRADS lexicon proved useful in uniform mammography reporting and consistency in lesion classification.

\section{KEYWORDS}

Diagnostic Mammography, Breast Masses, ACR-BIRADS.

HOW TO CITE THIS ARTICLE: Rathi V, Patankar K. Assessment of the breast masses with diagnostic mammography and FNAC correlation. J. Evolution Med. Dent. Sci. 2016;5(51):3265-3271, DOI: 10.14260/jemds/2016/758

\section{INTRODUCTION}

In India, breast cancer is the second most common cancer (After cervical cancer) with an estimated 115, 251 new diagnosis.(1) It is also the second most common cause of cancer-related deaths with 53,592 breast cancer deaths in 2008.(2) The age-standardised incidence rate for breast cancer in India is 22.9 per 100,000 , one-third that of Western countries, but the mortality rates are disproportionately higher.(3,4) Recent data suggests that breast cancer is the most common cancer in metropolitan cities and is predicted to be the leading cancer in the coming decade.(5)

However, majority of breast neoplasms are benign.(5) which makes proper distinction between benign and malignant imperative to avoid unnecessary surgical interventions and procedures.

Financial or Other, Competing Interest: None.

Submission 09-05-2016, Peer Review 03-06-2016,

Acceptance 09-06-2016, Published 25-06-2016.

Corresponding Author:

Dr. Varsha Rathi,

Block 4, Type 5,

ESIS Doctors Staff Quarters,

Ganpat Jadhav Marg,

Worli, Mumbai-18,

Maharashtra.

E-mail: drvpr@hotmail.com

DOI: $10.14260 /$ jemds $/ 2016 / 758$
The major cause of concern is the locally advanced stage of the malignancy when it first becomes detected.(6,7) This is alarming and makes case for a sound diagnostic technique for early detection. There is no organized, systematic (or government-funded) population-based screening program for breast cancer in India.(8,9) Evidence from developed countries shows that mammography reduces breast cancer mortality in women aged 50-69 years.(10) which has led to populationbased screening programs for older women in developed regions of the world such as Europe, North America, Australia and Japan.(11) In India, breast cancer incidence peaks before the age of 50 years and a recent review of the evidence.(12) in younger women (Aged 39-49 years) based on 8 trials conducted between 2001-2008, suggests that mammographic screening is also beneficial in this younger age group.

Since mammography is still in nascent stages in our country, we want to fill that data gap. In our study, we want to focus on mammography as a diagnostic as well as screening modality.

To bring reliability and reproducibility in mammography reporting American College of Radiology has devised the Breast Imaging Reporting and Data system (ACR BI-RADS). In our study, we referred the $4^{\text {th }}$ edition of ACR BI-RADS. 


\section{AIMS AND OBJECTIVES}

1. Characterizing the breast masses with mammography.

2. To study the utility of ACR BI-RADS Lexicon in describing and categorizing breast masses.

3. Assessing the efficacy of diagnostic mammography in correlation to cytopathology.

\section{MATERIALS AND METHODS}

This prospective, hospital based study of "Assessment of breast masses using mammography and their FNAC correlation" was carried out at IGGMC, Nagpur, a tertiary care institute over a period of two years. The institute serves a large population and is a premier teaching institute with undergraduate and postgraduate teaching courses. The study protocol was duly approved by the Institutional Ethical Committee.

\section{Study Population}

The study included 63 patients with breast lumps who were referred to the Department of Radiodiagnosis of our institute for mammography. Patients less than 30 years, although not in standard mammography protocol, also underwent mammography when advised by Surgery Department.

\section{Study Selection Criteria}

All patients of all ages referred for imaging evaluation with complaints of breast lumps and related symptoms were included in the study. However, patients with mastitis or those with ulcerated/fungating masses were excluded. Patients with previous $\mathrm{h} / \mathrm{o}$ breast cancer were only evaluated for the other side.

\section{Mammography Protocol}

1. Requisition form requesting for Breast mammography.

2. Detailed clinical history along with clinical examination findings were recorded.

3. The procedure was explained to the patient in detail and informed, written and valid consent as per the proforma was taken.

4. Patient were evaluated with the help of Allengers 4035 Venus Mammography Unit.

5. Standard mammography views were taken: Craniocaudal (CC) view and Mediolateral Oblique (MLO). Whenever needed sonography was also done.

6. Reporting of mammogram was done using BIRADS.

7. Later on biopsy/FNAC of the lesions was done to confirm the findings of mammography.

Statistical software STATA version 13.1 was used for statistical analysis and percentages were computed to present qualitative response variables like presenting complaints of breast lump and nipple discharge, BI-RADS mammogram findings and biopsy (FNAC/Trucut/excision) findings. Analysis was performed to compute sensitivity, specificity, accuracy, positive and negative predictive values of BI-RADS mammogram in the diagnosis of breast cancer on the basis of biopsy (FNAC/Trucut/excision) findings as gold standard.

\section{RESULTS}

\begin{tabular}{|l|c|c|c|c|}
\hline & FNAC Benign/Unremarkable & Percentage & FNAC Malignant & Percentage \\
\hline Total no. of Patients: 63 & 44 & 69.84 & 19 & 30.15 \\
\hline Total no. of Lesions: 70 & 51 & 72.85 & 19 & 27.14 \\
\hline \multicolumn{4}{|c|}{$\begin{array}{c}\text { Table 1: Distribution of Lesions into Benign and Malignant } \\
\text { According to Cytopathology Results (N = 70 Lesions in 63 Patients) }\end{array}$} \\
\hline
\end{tabular}

\section{Six Patients had more than One Lesion}

- In our study, we evaluated a total of 63 patients in the age group of 18-65 years, 3 males and 60 females.

- Mean age of patients in the benign and malignant category was $40.02 \pm 11.23$ years and $50.10 \pm 11.71$ years respectively.

- Lump (85.71\%) was the most common clinical symptom; 3 patients with past h/o cancer who had come for screening of uninvolved breast were included too.

- $\quad$ Bilateral cases were $2.27 \%$ of total. Left side (59.09\%) was more affected than right side (38.64\%).

- Superolateral+axillary tail quadrant was most commonly affected in both benign (47.06\%) and malignant (52.94\%) lesions.

- Infiltrating ductal carcinoma $(12 / 19,63.15 \%)$ and fibroadenoma $(21 / 51,41.17 \%)$ were the most common malignant and benign lesions respectively.

\begin{tabular}{|c|c|c|c|c|}
\hline BI-RADS Category & No. of Patients & Percentage & Cytopathology Benign & Cytopathology Malignant \\
\hline 1 & 19 & $30.15 \%$ & 17 & 2 \\
\hline 2 & 6 & $9.52 \%$ & 6 & 0 \\
\hline 3 & 21 & $33.33 \%$ & 20 & 1 \\
\hline 4 & 12 & $19.04 \%$ & 1 & 5 \\
\hline 5 & 5 & $7.93 \%$ & 0 & $\mathbf{4 4}$ \\
\hline Total & $\mathbf{6 3}$ & $\mathbf{1 0 0 \%}$ & $\mathbf{4}$ & \\
\hline \multicolumn{7}{|c|}{ Table 2: Mammographic ACR BI-RADS Classification (N=63) } \\
\hline
\end{tabular}

\begin{tabular}{|c|c|c|c|}
\hline \multirow{2}{*}{ Mammographic Classification } & FNAC & Total \\
\cline { 2 - 4 } & Benign & Malignant & 46 \\
\hline Benign (BI-RADS 1, 2, 3) & $43(\mathrm{TN})$ & $3(\mathrm{FN})$ & 17 \\
\hline Malignant (BI-RADS 4,5) & $1(\mathrm{FP})$ & $16(\mathrm{TP})$ & \\
\hline \multicolumn{2}{|c|}{ Table 3: Mammographic Versus FNAC Classification of Lesions (N=63) } \\
\hline
\end{tabular}




\begin{tabular}{|l|c|c|}
\hline Sensitivity & $=\mathrm{TP} /(\mathrm{TP}+\mathrm{FN})$ & $84.21 \%$ \\
\hline Specificity & $=\mathrm{TN} /(\mathrm{TN}+\mathrm{FP})$ & $97.73 \%$ \\
\hline Positive Predictive Value (PPV) & $=\mathrm{TP} / \mathrm{TP}+\mathrm{FP}$ & $94.12 \%$ \\
\hline Negative Predictive Value(NPV) & $=\mathrm{TN} / \mathrm{TN}+\mathrm{FN}$ & $93.48 \%$ \\
\hline Accuracy & $=(\mathrm{TP}+\mathrm{TN}) / \mathrm{TOTAL}$ & $93.65 \%$ \\
\hline Table 4: Table Sensitivity, Specificity, PPV, NPV and Accuracy of Mammography using ACR BI-RADS.22 for Malignant Lesions \\
\hline
\end{tabular}

\begin{tabular}{|c|c|c|c|c|c|c|}
\hline $\begin{array}{c}\text { BI-RADS } \\
\text { Category }\end{array}$ & $\begin{array}{c}\text { No. of } \\
\text { Patients }\end{array}$ & Percentage & $\begin{array}{c}\text { Cytopathology } \\
\text { Benign }\end{array}$ & $\begin{array}{c}\text { Cytopathology } \\
\text { Malignant }\end{array}$ & NPV & PPV \\
\hline 1 & 19 & $30.15 \%$ & 17 & 2 & $09.47 \%$ & $0 \%$ \\
\hline 2 & 6 & $9.52 \%$ & 6 & 1 & $100 \%$ & $0 \%$ \\
\hline 3 & 21 & $33.33 \%$ & 20 & 11 & $95.23 \%$ & $0 \%$ \\
\hline 4 & 12 & $19.04 \%$ & 1 & 5 & $0 \%$ & $91.66 \%$ \\
\hline 5 & 5 & $7.93 \%$ & 0 & $\mathbf{1 9}$ & - & - \\
\hline Total & $\mathbf{6 3}$ & $\mathbf{1 0 0 \%}$ & $\mathbf{4 4}$ & \multicolumn{2}{c}{} \\
\hline \multicolumn{7}{r|}{} \\
\hline
\end{tabular}

In our study BI-RADS categories 1, 2 and 3 have been considered as negative studies for malignancy. Hence, from this point of view, NPV of categories 1, 2 and 3 is $89.47 \%$, $100 \%$ and $95.23 \%$ respectively. BI-RADS categories 4 and 5 have been considered positive studies for malignancy and have positive predictive value of $91.66 \%$ and $100 \%$ respectively.

- Sensitivity and PPV is maximum in almost entirely fatty breasts (<25\% glandular): $47.36 \%$ and $50 \%$ respectively. Heterogeneously dense breasts and extremely dense breasts were related to false negative cases.

- Amongst mass shape descriptors in BI-RADS Lexicon, irregular shaped masses showed high positive predictive value for malignancy and were categorized as BI-RADS 4 or 5 . The sensitivity of irregular shape was highest$73.68 \%$. Round and oval shaped lesions were always benign.

- Amongst the mass margin descriptors in BI-RADS Lexicon, indistinct margin had highest sensitivity followed by spiculated margins, $36.84 \%$ and $21.05 \%$ respectively. However, both indistinct and spiculated margins had positive predictive value of $100 \%$ for malignancies and favoured BIRADS 4 or 5 categorization. Circumscribed and obscured margins had 0\% predictive value for malignancy, that is circumscribed margins had a NPV of $100 \%$ for malignancy.

- Amongst the mass density descriptors in BI-RADS, in our study, high density lesions had highest PPV for malignancy-44.82\%. Sensitivity for malignancy was maximum for high-density lesions: $68.42 \%$.

- In our study, calcification was present as the main finding in four cases. Two cases showed coarse amorphous calcification, which in the BI-RADS Lexicon comes under category of Intermediate concern, suspicious calcification. Both the cases showed histopathological diagnosis of ductal carcinoma in situ. Thus, coarse amorphous calcification had a PPV for malignancy of $100 \%$ and sensitivity of $10.52 \%$ (2/19) for malignancy. Two cases, which were found to be benign/unremarkable on cytopathology showed findings of linear large rods like calcifications and lucent-centered calcifications in regional distribution. Thus, the BI-RADS Lexicon for benign calcifications was found to have NPV of $100 \%$ for malignancy.
Amongst cases in which calcification was present as an associated finding, pleomorphic calcification in clustered distribution was present as an associated finding in two cases of carcinoma. Thus, PPV of pleomorphic calcifications for malignancy comes to $100 \%$.

- No patient in our study presented with architectural distortion as the main finding. However, as an associated finding, it was present in eight patients. Seven of these patients were found to have malignant lesions; one benign lesion was infected galactocele.

- We had two studies of focal asymmetry. One of them was due to infiltrating ductal ca (This was categorized as BIRADS 3-hence false negative), the other was due to tubercular abscess. Thus, asymmetry does not have a good sensitivity for malignancy. It is the finding with the lowest cancer yield at mammography.

\section{DISCUSSION}

The present prospective hospital based study entitled "Assessment of breast masses with mammography and their FNAC correlation" was carried over at our Institute Department of Radiology, IGGMC, Nagpur, during the period from December 2012 to November 2014.

\section{Demographic Criteria}

Amongst 63 patients evaluated, 3 were males and 60 were females. Thus males are affected by diseases of breast too.

The most common age group for malignant lesions was 41 50 years. This shows alarming trend of breast cancer presenting early in our population compared to that in western population. The earliest age presenting with atypia in our study has age of 27 years.

\section{ACR BI-RADS Categorization.13,14}

Devised with the intention of standardizing mammography reporting and providing guidance to mastologists on the probability of malignancy. This system comprises a specific vocabulary for describing each lesion and as a report conclusion, the study result is classified into categories ranging from 0 to 6 according to the degree of suspicion for malignancy.

In our study, 19 patients were categorized in BI-RADS 1 , out of which 17 patients had benign results; two had malignant results on cytopathology. 
BI-RADS 2 had six patients, all of whom were having benign lesions on cytopathology. BI-RADS 3 had twenty-one patients with just one having malignant lesion and the rest benign on cytopathology.

BI-RADS 4 had twelve patients, out of which eleven were having malignant lesions on cytopathology. BI-RADS 5 had five patients, all of which were having malignant lesions on cytopathology.

Mammographic versus Cytopathological Classification of Lesions: Sensitivity, Specificity, PPV, NPV and Accuracy of Mammography using ACR-BIRADS.

Most of these lesions of category 1 had cytopathological diagnosis of fibrocystic disease. While tiny cysts were identified on ultrasound, they could not be discerned on mammography. This proves that for such lesions mammography had good NPV, but cannot be specific about the diagnosis and cannot identify small cysts.

Amongst the three false negative cases, one was a 27 years-old female with heterogeneously dense breast with proliferative breast disease mild atypia. Another was also a 32 yrs. female with heterogeneously dense breast and infiltrating ductal carcinoma. The third case was again a female with heterogeneously dense breast with focal asymmetry and infiltrating duct carcinoma. Thus, dense breasts are a common thread in cause for false negative cases.

The false positive case was due to $b / l$ retroareolar abscesses in a 45 yrs. old female. Our results are comparable with others in literature and mammography has good sensitivity, specificity, positive predictive value, negative predictive value and accuracy. $(15,16,17,18)$

The number of biopsies performed for benign lesion can be reduced by using BI-RADS categories, as it has a good PPV.

\section{Composition of Breast}

Sensitivity and PPV of mammography is maximum in almost fatty breasts $(<25 \%$ glandular tissue $)$.

The false negative cases were related to heterogeneously dense breasts or extremely dense breasts respectively.

\section{Sensitivity of BI-RADS Lexicon for Malignancy}

- After studying the various BIRADS Lexicon descriptors in our study of all BIRADS Lexicon descriptors, irregular shape has highest sensitivity for malignant mass at $73.68 \%$ followed by high-density mass at $68.42 \%$, indistinct mass margin at $36.84 \%$ and spiculated mass margin at $21.05 \%$. Irregular shape had a positive predictive value of $85.71 \%$ for malignancy. Indistinct and spiculated margins had $100 \%$ positive predictive value for malignancy. Our study is in agreement with other similar studies; however, since we had greater number of lesions with indistinct margins its sensitivity was more than that for spiculated margins. $(19,20,21,22)$

- High density mass had positive predictive value of $44.82 \%$ for malignancy.

- Calcifications had low sensitivity of $10.52 \%$ as main finding. Amongst calcifications, coarse amorphous calcification had a PPV for malignancy of $100 \%$ for malignancy. Two cases which were malignant, presented as a spiculated mass with pleomorphic calcifications as associated finding. Thus, PPV for these pleomorphic calcifications was $100 \%$. In our study, we had few cases with calcification.

This could be one of the reasons overall sensitivity of calcification descriptors was less. We attribute this to the fact that majority of patients in our set-up presented in a late stage. However, the cases related to calcification descriptors are in confirmation with BI-RADS Lexicon and in most cases help to determine the malignancy or benignity of lesions accurately.

- No patient in our study presented with architectural distortion as the main finding. However, as an associated finding, it was present in eight patients. Seven of these patients were found to have malignant lesions; one benign lesion was infected galactocele. Sensitivity of architectural distortion as an associated finding comes to $36.84 \%$. It has a low prevalence, but is highly predictive of invasive cancer at screening and diagnostic examination.

\section{Additional Findings}

In our study the finding of skin retraction was associated with 2 lesions, both of which were malignant. Axillary lymphadenopathy was present in four patients, two of which had malignant lesions. Nipple retraction was associated with one case of malignancy. Additional findings do not affect the final BI-RADS categorization; however, their high PPV is a good reasoning for their incorporation as a component influencing BIRADS.

\section{Presentation of Malignancy}

In our study, mass was the most common form in which malignancies presented with $63.15 \%$ followed by mass with calcification (10.52\%) and just calcifications (10.52\%) as main finding.

\section{Limitations}

It is technically difficult to do mammography of small breasts and dense glandular breasts hinder visualisation of lesions. Fibrocystic lesions in smaller size are not visualised as mass on mammography. Not all patients were able to tolerate the pain related compression. In such cases, ultrasound comes in handy, a problem solving modality.

\section{CONCLUSIONS}

Mammography is highly sensitive and accurate in detection and characterization of breast masses especially the malignant masses. The descriptors of the standardized mammographic ACR-BIRADS Lexicon are extremely useful in characterization and categorization of focal breast masses and provide consistency of lesion classification.

\section{Recommendations}

There is an alarming trend of breast cancer occurring early in Indian females (In their forties) and breast cancer is poised to become number one cancer amongst females. For this it is essential that we have screening and diagnostic mammography as a part of National programs. To overcome fallacies of mammography especially in dense breasts, it should be combined with sonography. 
Case Studies

Case 1
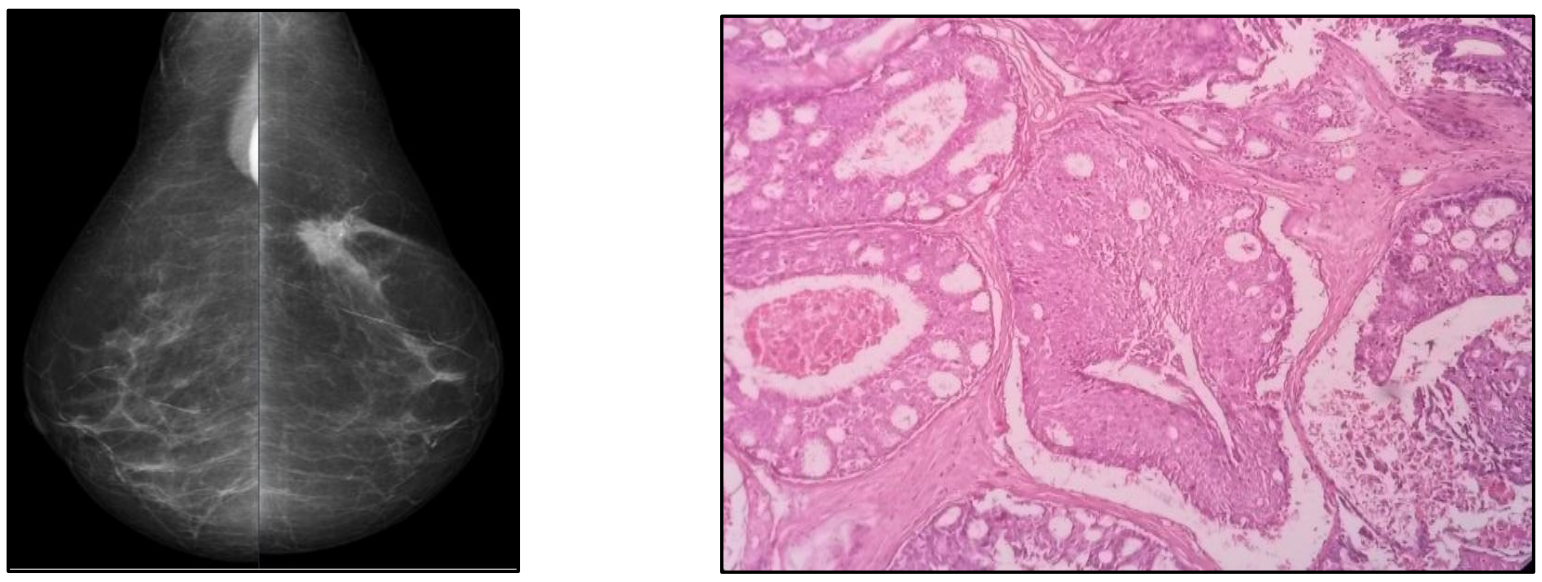

Breast Composition: Almost Entirely Fat L-MLO View in Superolateral Quadrant shows an Irregular, Spiculated, High Density Mass with Architectural Distortion and Associated Pleomorphic Calcification: BI-RADS 5 Cytopathology: Infiltrating Ductal Carcinoma

Case 2
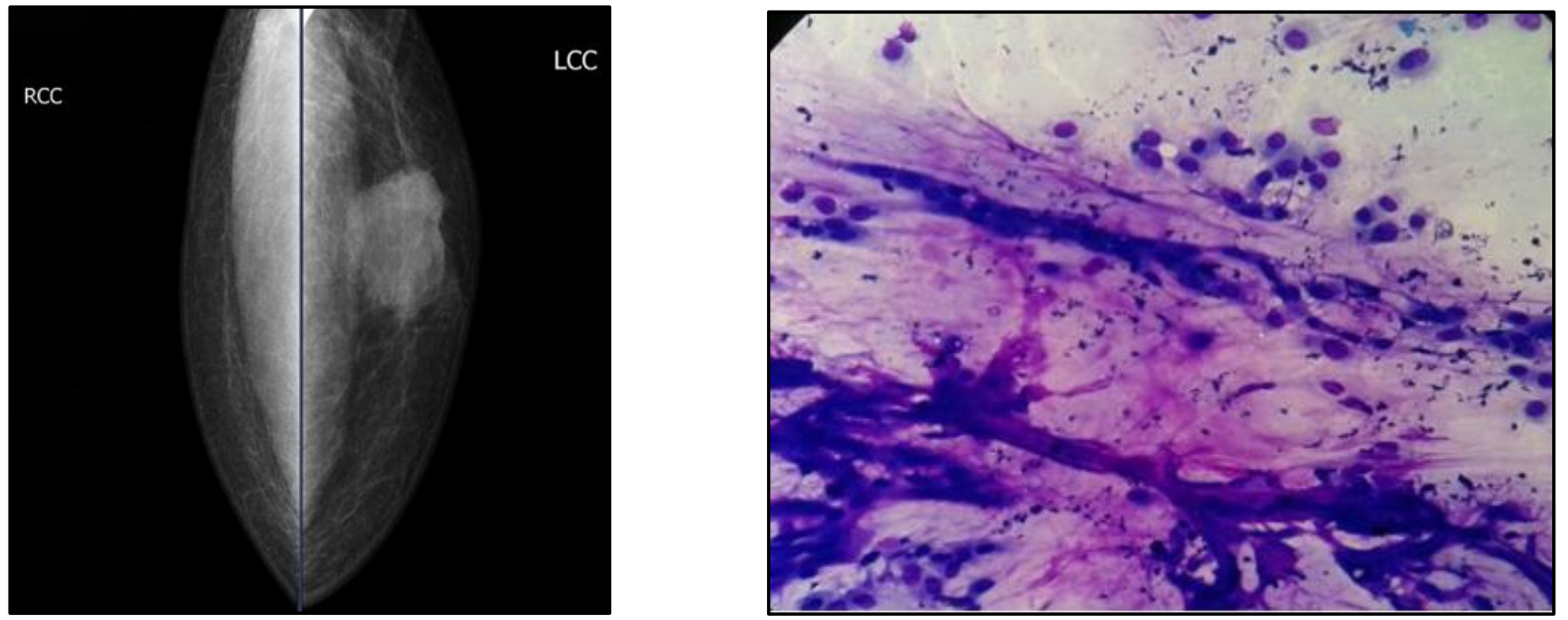

Breast Composition: Almost fatty in a Male Breast, LCC, High Density Mass with Lobular Shape and Indistinct Margin in Left Retroareolar Region: BI-RADS 4 Cytopathology: Mucinous Carcinoma

Case 3
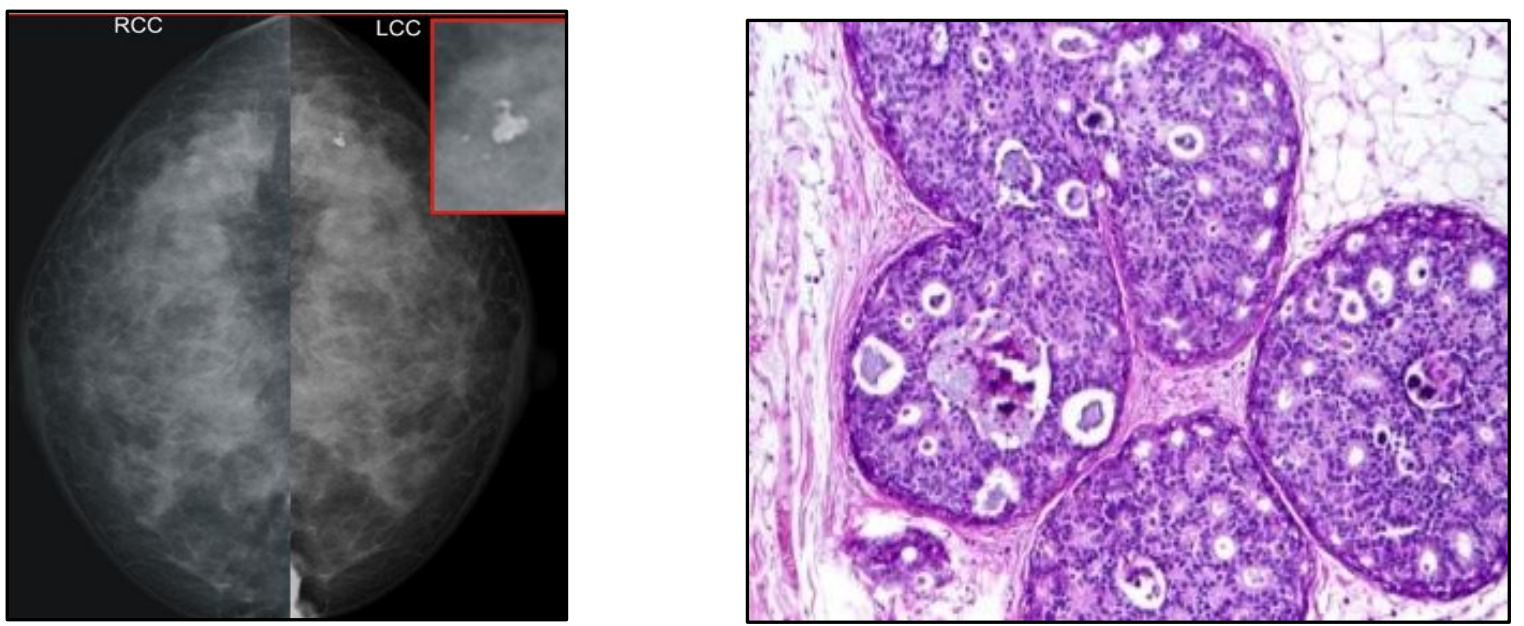

Breast Composition: Heterogeneously Dense Breast in LCC, Superolateral Quadrant Posteriorly, shows Grouped, Coarse Amorphous Calcifications: BI-RADS 4 Cytopathology: Ductal Carcinoma-In-Situ 
Case 4
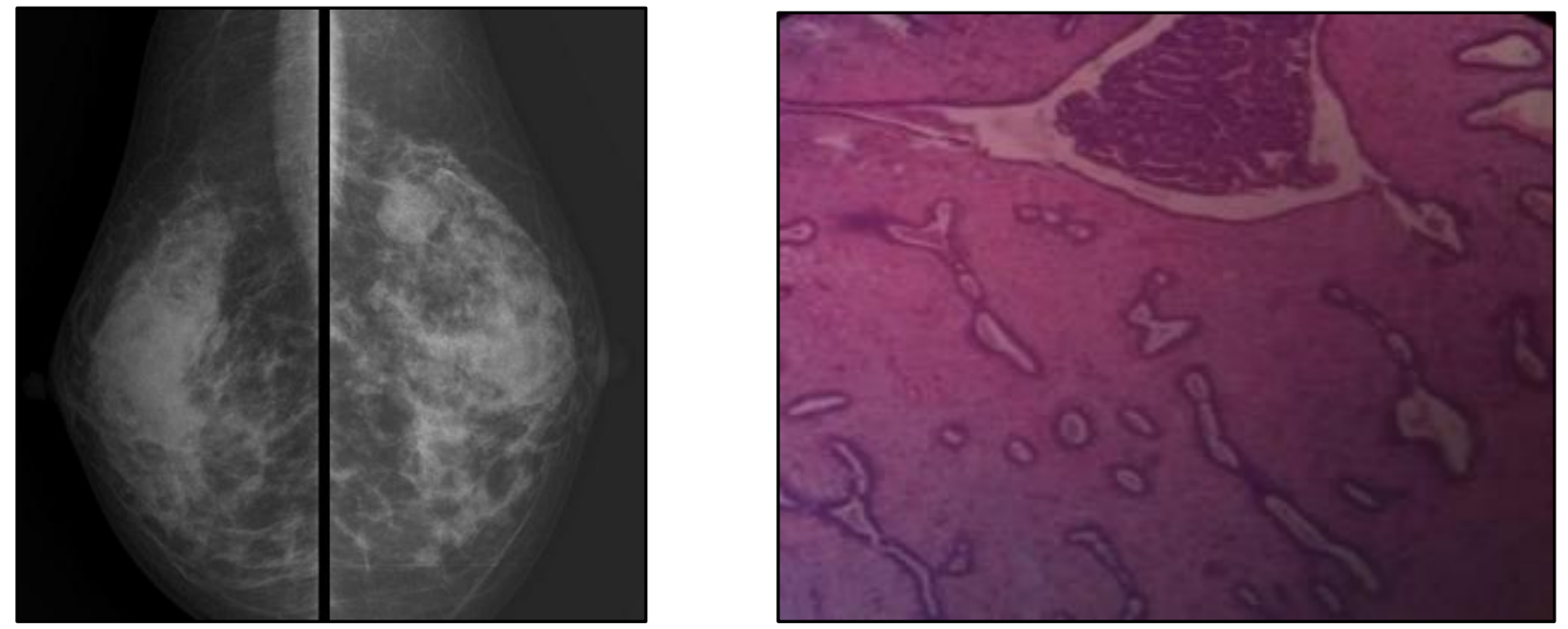

Breast Composition: Scattered Fibroglandular Densities (Approximately 25-50\% Glandular) In L-MLO View, Posteriorly, Round, Circumscribed, Isodense Mass Noted in Superolateral Quadrant: BI-RADS 3 Cytopathology: Fibroadenoma

Case 5
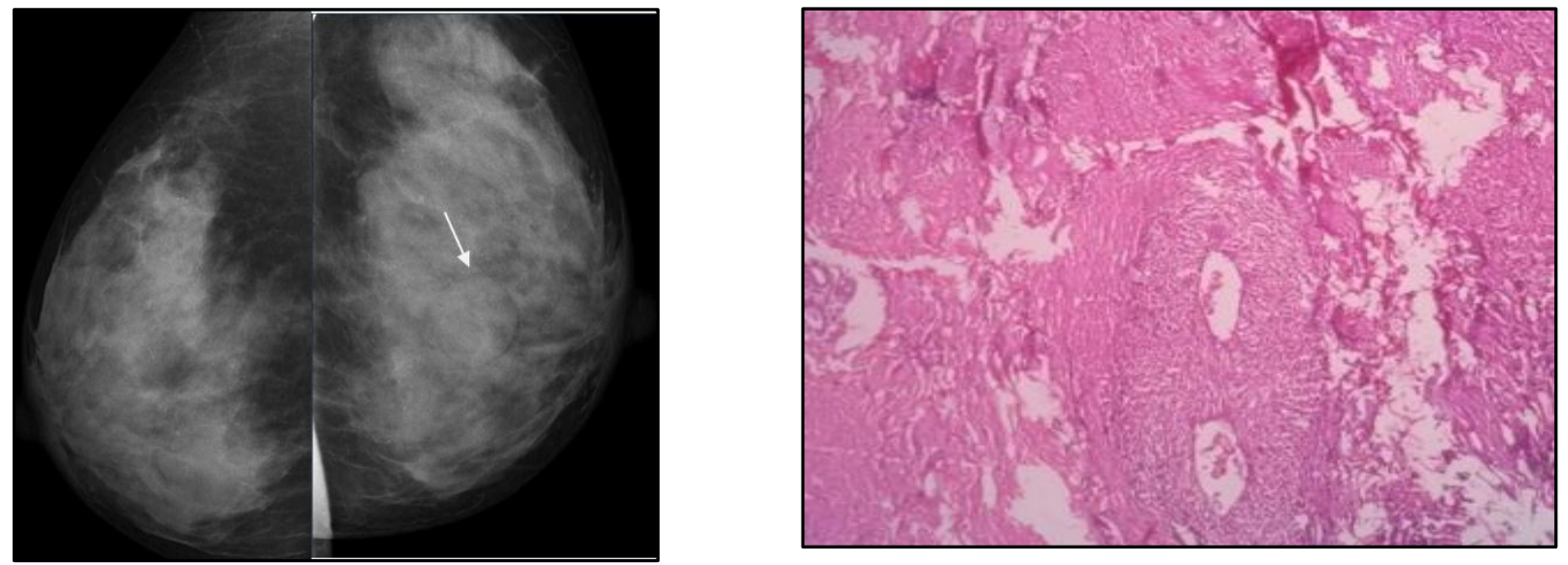

Breast Composition: Dense Breast in LCC, Inferomedial Quadrant, Round, Circumscribed, Isodense Lesion: BIRADS 3 Cytopathology: Fibrocystic Disease of Breast

Case 6
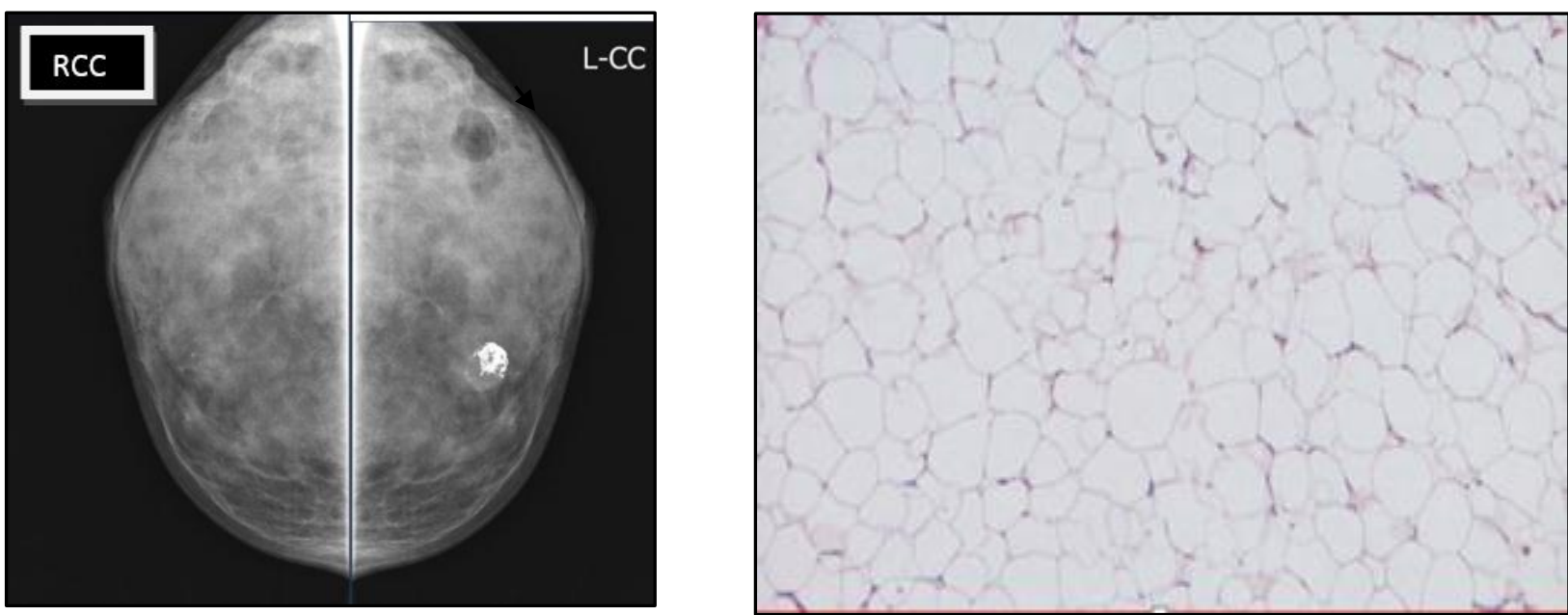

Breast Composition: Heterogeneously Dense Breast (51-75\% Glandular) in LCC View, in Superolateral Quadrant, in Middle Third of Breast, Fat Density Lesion Noted, and in Inferomedial Quadrant, in Middle Third of Breast, Oval, Isodense Lesion with Popcorn Calcification Noted: BI-RADS 2.

Cytopathology: Lipoma in Superolateral Quadrant and Calcified Fibroadenoma in Inferomedial Quadrant. 


\section{REFERENCES}

1. Asthana S, Chauhan S, Labani S. Breast and cervical cancer risk in India: an update. Indian J Public Health 2014;58(1):5-10.

2. Parkin DM, Bray F, Ferlay J, et al. Estimating the world cancer burden: globocan 2000. Int J Cancer 2001;94(2):153-6. doi:10.1002/ijc.1440.

3. Agarwal G, Ramakant P. Breast cancer care in India: the current scenario and the challenges for the future. Breast Care (Basel) 2008;3(1):21-7.

4. Agarwal G, Ramakant P, Forgach ER, et al. Breast cancer care in developing countries. World J Surg 2009;33(10):2069-76.

5. Kumar M, Ray K, Harode S, et al. The pattern of benign breast disease in rural hospital in India. East Central African J Surg 2010;15(2):59-64.

6. Advani S. Partner profile: cancer in India. INCTR News 2004;5:18.

7. Chopra R. The Indian scene. J Clin Oncol 2001;19:106-11.

8. Leong SP, Shen ZZ, Liu TJ, et al. Is breast cancer the same disease in Asian and Western countries? World J Surg 2010;34(10):2308-24.

9. Mittra I, Mishra GA, Singh S, et al. A cluster randomized controlled trial of breast and cervix cancer screening in Mumbai, India: methodology and interim results after three rounds of screening. Int J Cancer 2010;126(4): 976-84.

10. DeKoning HJ. Mammographic screening: evidence from randomised controlled trials. Ann Oncol 2003;14:1185-9.

11. Shapiro S, Coleman EA, Broeders M, et al. Breast cancer screening programmes in 22 countries: current policies administration and guidelines. International breast cancer screening network and the European network of pilot projects for breast cancer screening. Int J Epidemiol 1998;27(5):735-42.

12. Nelson HD, Tyne K, Naik A, et al. Screening for breast cancer: an update for the US preventive services task force. Ann Intern Med 2009;151(10):727-37, W237-42.
13. American college of radiology. Breast imaging reporting and data system ${ }^{\circledR}$ (BI-RADS ${ }^{\circledR}$ ) Restonva: American college of radiology 1992.

14. American college of radiology. Breast imaging reporting and data system ${ }^{\circledR}$ (BI-RADS $\left.®\right)$ 4th edn. Reston, Va: American college of radiology 2003.

15. Al-Mulhim AS, Sultan M, Al-Mulhim FM, et al. Accuracy of the triple test in the diagnosis of palpable breast masses in Saudi females. Ann Saudi Med 2003;23(3-4):158-61.

16. Hirunpat S, Tanomkiat W, Khojarern R, et al. Accuracy of the mammographic report category according to birads. J Med Assoc Thai 2005;88(1):62-5.

17. Arsalan F, Subhan A, Rasul S, et al. Sensitivity and specificity of bi-rads scoring system in carcinoma of breast. J Surg Pak 2010;15(1):38-43.

18. Fatima N, Zaman M, Qadeeruddin, et al. Accuracy of mammography and ultrasound for detecting breast cancer at a breast care clinic in Karachi, Pakistan. Journal of Biomedical Graphics and Computing 2011;1(1):44-50.

19. Liberman L, Abramson AF, Squires FB, et al. The breast imaging reporting and data system: positive predictive value of mammographic features and final assessment categories. American Journal of Roentgenology 1998;171(1):35-40.

20. Nascimento JHR, Silva VD, Maciel AC. Accuracy of mammographic findings in breast cancer: correlation between bi-rads classification and histological findings. Radiol Bras 2010;43(2):91-6.

21. Woods RW, Sisney GS, Salkowski LR, et al. The mammographic density of a mass is a significant predictor of breast cancer. Radiology 2011;258(2):417-25.

22. Tamaki K, Ishida T, Miyashita M, et al. The mammographic density of a mass is a significant predictor of breast cancer. Radiology 2011;103(3):472-6. 